\title{
Avaliação da capacidade funcional dos idosos residentes em uma instituição de longa permanência
}

\author{
Evaluation of the functional capacity of elderly residents in a long stay institution
}

\author{
Evaluación de la capacidad funcional de ancianos residentes en una institución de larga \\ duración
}
Francisca Souza Santos Dias ${ }^{1 *}$, Clara Cynthia Melo e Lima ${ }^{1}$, Patrícia de Sousa Fernandes Queiroz ${ }^{2}$, Tatiana Fróes Fernandes ${ }^{3}$.

\section{RESUMO}

Objetivo: Avaliar o grau de independência dos idosos de uma instituição de longa permanência na realização das atividades básicas da vida diária e correlacionar com o seu perfil sócio-demográfico. Métodos: Trata-se de estudo transversal descritivo, com abordagem quantitativa. O estudo foi conduzido com 24 idosos, de ambos os sexos, residentes em uma instituição de longa permanência em uma cidade de Minas Gerais. Os dados foram coletados por aplicação de questionário sócio-demográfico e da escala de Katz, para a mensuração das Atividades Básicas de Vida Diária. Os dados obtidos foram analisados por estatística descritiva. O estudo foi aprovado por Comitê de Ética em Pesquisa. Resultados: A amostra, com idade entre 60 e 95 anos, apresentou maioria masculina (54,2\%), solteira (71\%), analfabeta (83\%), que não possui filhos (71\%). Na avaliação do desempenho das atividades básicas de vida diária, constatou-se que a maioria dos idosos era independente $(54,2 \%)$, seguida dos dependentes $(37,5 \%)$ e dos parcialmente dependentes $(8,3 \%)$. Dentre os seis itens avaliados, apenas em vestir-se, a maioria foi dependente (54,2\%). Conclusão: Conclui-se que na avaliação da capacidade funcional, os homens apresentaram dados de independência mais positivos que as mulheres, sendo que a maioria dos independentes possuía idade inferior a 80 anos.

Palavras-chave: Envelhecimento, Atividades cotidianas, Instituição de longa permanência para idosos.

\section{ABSTRACT}

Objective: Assess the degree of independence of the elderly in a long-term institution in carrying out basic activities of daily living and correlate with their socio-demographic profile. Methods: This is a descriptive cross-sectional study, with a quantitative approach. The study was conducted with 24 elderly people, of both genders, living in a long-term institution in a city in Minas Gerais. Data were collected by applying a socio-demographic questionnaire and the Katz scale, to measure the Basic Activities of Daily Living. The data obtained were analyzed using descriptive statistics. The study was approved by the Research Ethics Committee. Results: The sample, aged between 60 and 95 years, had a male majority (54.2\%), single $(71 \%)$, illiterate (83\%), who did not have children (71\%). In assessing the performance of basic activities of daily living, it was found that the majority of the elderly were independent $(54.2 \%)$, followed by dependents (37.5\%) and partially dependent (8.3\%). Among the six items evaluated, only in dressing, most were dependent $(54.2 \%)$. Conclusion: It was concluded that in the assessment of functional capacity, men presented more positive data of independence than women, and most of the independents were below the age of 80 years.

Keywords: Aging, Activities of daily living, Homes for the aged.

\section{RESUMEN}

Objetivo: Valorar el grado de independencia de las personas mayores en una institución de larga duración en el desempeño de las actividades básicas de la vida diaria y correlacionarlo con su perfil sociodemográfico. Métodos: Se trata de un estudio descriptivo transversal, con enfoque cuantitativo. El estudio se realizó con 24 personas mayores, de ambos sexos, residentes en una institución de larga duración en una ciudad de Minas Gerais. Los datos fueron recolectados mediante la aplicación de un cuestionario sociodemográfico y la escala de Katz, para medir las Actividades Básicas de la Vida Diaria. Los datos obtenidos se analizaron mediante estadística descriptiva. El estudio fue aprobado por el Comité de Ética en Investigación. Resultados: La muestra, con edades comprendidas entre 60 y 95 años, fue mayoritariamente masculina $(54,2 \%)$, soltera $(71 \%)$, analfabeta $(83 \%)$, que no tuvo hijos $(71 \%)$. Al evaluar el desempeño de las actividades básicas de la vida diaria, se encontró que la mayoría de los ancianos eran independientes (54,2\%), seguidos de los dependientes $(37,5 \%)$ y parcialmente dependientes $(8,3 \%)$. De los seis ítems evaluados, solo en vestimenta, la mayoría eran dependientes (54,2\%). Conclusión: Se concluyó que en la evaluación de la capacidad funcional los hombres presentaron datos más positivos de independencia que las mujeres, y la mayoría de los independientes tenían menos de 80 años.

Palabras clave: Envejecimiento, Actividades cotidianas, Hogares para ancianos.

\footnotetext{
${ }^{1}$ Instituto Federal do Norte de Minas Gerais (IFNMG), Araçuaí - MG. *E-mail: francisca_souzasantos@yahoo.com.br

2 Instituto Federal do Norte de Minas Gerais (IFNMG), Januária - MG.

3 Universidade Estadual de Montes Claros (UNIMONTES), Montes Claros - MG.
} 


\section{INTRODUÇÃO}

O aumento da proporção de idosos é um fenômeno global que, no Brasil, em virtude da evolução tecnológica na área da saúde, bem como o declínio das taxas de fecundidade, provocou um sensível aumento da expectativa de vida da população idosa, ocasionando modificações mais rápidas e profundas quando comparado com países desenvolvidos (GÜTHS JFS, et al., 2017). Trata-se de um processo complexo que determina mudanças nas dimensões biológica, social e psicológica do sujeito, acontecendo de maneira heterogênea, uma vez que cada indivíduo envelhece de acordo com sua genética, hábitos de vida e meio onde está inserido (SILVA RS, et al., 2019).

Nesse sentido, envelhecer é um processo dinâmico e inerente a todos os seres humanos, acarretando alterações fisiológicas, psicológicas e socioculturais, podendo ser definidas como um processo senescente, próprio do envelhecimento, ou caracterizado como senilidade, quando se trata de quadro patológico (LEITE AK, et al., 2020).

As mudanças ocasionadas pelo envelhecimento, associadas aos abusos da juventude, condições e hábitos de vida e desuso, podem ser desencadeadoras de complicações características dessa época da vida humana. Em sua maioria, essas complicações são definidas por processos fisiológicos e/ou patológicos crônicos, podendo ser incapacitantes ou geradoras de uma condição de dependência (LEAL RC, et al., 2019).

Mesmo com o cuidado familiar ainda predominante, observa-se aumento na demanda por cuidado formal de longa duração, tendo em vista que com o processo de envelhecimento muitas vezes associado à presença de doenças crônico-degenerativas propiciando situações de vulnerabilidade para o idoso (SOARES PPB, et al., 2015; LEAL RC, et al., 2019). Neste sentido, as Instituições de Longa permanência para Idosos (ILPI) mostram-se como opção para esse tipo de cuidado e como alternativa para atender às necessidades sociais da sociedade moderna. De acordo com a RDC nำ 283/05 da ANVISA, todas as ILPI (públicas, filantrópicas ou privadas) são de caráter residencial e se destinam a moradia coletiva de pessoas com idade igual ou superior a 60 anos que possuem ou não suporte familiar (ANVISA, 2005).

A crescente procura por essas instituições está relacionada às alterações nas configurações e arranjos familiares que implicam na diminuição da oferta de cuidado no contexto familiar (CARRARA BS e ESPIRITO SANTO PMF, 2016). Outro fator que interfere nessa demanda é a incapacidade funcional que é definida como o conjunto de competências físicas e mentais essenciais para executar, sem auxílio, as atividades da vida diária. Para os idosos, essa questão significa que estão aptos para desenvolver atividades e tomar decisões do seu cotidiano (KAGAWA CA e CORRENTE JE, 2015). A incapacidade funcional reduz a autonomia na execução de atividades básicas, assim, avaliar o desempenho das atividades de vida diária pelos idosos é relevante, pois pode fornecer suporte para nortear uma assistência de qualidade dos serviços de saúde (GONÇALVES MJC, et al., 2015).

Para a avaliação funcional do idoso, é amplamente utilizada a escala de Katz. O Índice de Katz foi desenvolvido na década de 1960 e consta de seis itens que medem o desempenho do indivíduo na realização das atividades básicas da vida diária, contendo questões sobre alimentar-se, banhar-se, continência, transferência, vestir-se e utilizar o banheiro (KATZ S, et al, 1963). A realização de avaliações sistemáticas com idosos que residem em instituições de longa permanência torna-se necessário ao considerar as peculiaridades do processo de envelhecimento humano relacionados aos aspectos da institucionalização. Nesse contexto, é de extrema relevância a avaliação da capacidade funcional dos idosos, de modo a planejar a assistência de maneira adequada e encontrar formas de melhorar a qualidade de vida de idosos residentes em ILPI, visando garantir a promoção da saúde e prevenção da incapacidade funcional (AZEVEDO LM, et al., 2017).

Considera-se que a realização das atividades básicas é um indicativo de qualidade de vida para o idoso, sendo um parâmetro legítimo para concretizar uma avaliação. O estudo tem por objetivo avaliar o grau de independência dos idosos de uma instituição de longa permanência na realização das atividades básicas da vida diária e correlacionar com o seu perfil sócio-demográfico. 


\section{MÉTODOS}

Trata-se de um estudo transversal, descritivo, com abordagem quantitativa. A pesquisa foi realizada em uma instituição de longa permanência para idosos, em uma cidade de Minas Gerais. Essa instituição é de caráter filantrópico, sem fins lucrativos, sendo regida por um estatuto e legislação brasileira. Tendo como principal objetivo dar assistência à velhice desamparada, com uma população de 35 indivíduos de ambos os sexos.

A coleta de dados foi realizada no período de janeiro a fevereiro de 2020 . Participaram da pesquisa $68,6 \%$ (24) dos residentes que atenderam aos seguintes critérios de inclusão: idade igual ou superior a 60 anos, que aceitaram participar da pesquisa, que possuíam condições cognitivas para responder ao questionário ou a presença de um cuidador para tal, e que residiam na instituição no momento da coleta.

Os dados foram obtidos por meio de entrevista face a face, realizada na instituição pela pesquisadora principal. Os fatores sociodemográficos e econômicos foram obtidos através de questionário semiestruturado incluindo as variáveis: idade, sexo, escolaridade, situação conjugal, presença de filhos e recebimento de visitas.

Para a avaliação do grau de independência para atividades de vida diária, foi utilizada a escala de Katz. O Index de Independência nas Atividades de Vida Diária ou Escala de Independência em Atividades da Vida Diária foi desenvolvido por Sidney Katz, trata-se de um instrumento de avaliação funcional bastante utilizado em estudos gerontológicos em nível nacional, bem como internacional. Segundo essa escala, os idosos são classificados como independentes quando desenvolvem a atividade sem supervisão, orientação ou qualquer tipo de auxílio direto. No Brasil, por muito tempo a tradução livre da Escala de Katz foi amplamente utilizada. Contudo, tendo em vista a importância do conhecimento do desempenho nas atividades da vida diária na avaliação de idosos e a relevância da utilização de instrumentos adaptados para a nossa cultura, Lino VTS, et al. (2008) realizaram a adaptação transcultural da Escala de Independência em Atividades da Vida Diária para a língua portuguesa (Quadro 1).

Quadro 1 - Adaptação transcultural da Escala de Independência em Atividades da Vida Diária.

\begin{tabular}{|c|c|c|}
\hline Atividade & Independentes & Dependentes \\
\hline Banhar-se & $\begin{array}{l}\text { Banham-se completamente ou necessitam de } \\
\text { auxílio somente para lavar uma parte do } \\
\text { corpo, como: as costas, genitais ou uma } \\
\text { extremidade incapacitada. }\end{array}$ & $\begin{array}{l}\text { Não são capazes de se banhar } \\
\text { sozinhos; }\end{array}$ \\
\hline Vestir-se & $\begin{array}{l}\text { Pega as roupas no armário e vesti-se, } \\
\text { compreendendo roupas íntimas, externas e } \\
\text { cintos. Calçar sapatos foi excluído da } \\
\text { avaliação. }\end{array}$ & $\begin{array}{l}\text { Requer auxílio para vestir-se ou } \\
\text { necessita ser completamente } \\
\text { vestido. }\end{array}$ \\
\hline Ir ao banheiro & $\begin{array}{l}\text { Dirige-se ao banheiro, entra e sai do mesmo, } \\
\text { arruma as próprias roupas, higieniza a área } \\
\text { genital sem ajuda, podendo ou não utilizar } \\
\text { algum suporte mecânico para desempenhar a } \\
\text { função. }\end{array}$ & $\begin{array}{l}\text { Requer ajuda para ir até o vaso } \\
\text { sanitário e usá-lo ou utilizam } \\
\text { "papagaios" ou "comadres". }\end{array}$ \\
\hline Transferência & $\begin{array}{l}\text { Senta-se e deita-se e/ou levanta-se da cama } \\
\text { ou cadeira sem ajuda, podendo utilizar } \\
\text { equipamentos ou suporte mecânico. }\end{array}$ & $\begin{array}{l}\text { Necessita de ajuda para sentar-se } \\
\text { e deitar-se e/ou levantar-se da } \\
\text { cama ou cadeira. Não faz uma ou } \\
\text { mais transferências. }\end{array}$ \\
\hline Continência & $\begin{array}{l}\text { Micção e evacuação } \\
\text { autocontroladas. }\end{array}$ & $\begin{array}{l}\text { Incontinência total ou parcial para } \\
\text { micção ou evacuação. Qualquer } \\
\text { tipo de controle externo ou uso } \\
\text { regular de fraldas. }\end{array}$ \\
\hline Alimentação & $\begin{array}{l}\text { Direciona a comida do prato à boca. O ato de } \\
\text { cortar os alimentos ou prepará-los é excluído } \\
\text { da avaliação. }\end{array}$ & $\begin{array}{l}\text { Requer qualquer assistência } \\
\text { pessoal. }\end{array}$ \\
\hline
\end{tabular}

Fonte: Lino VTS, et al, 2008. 
No índice, foi contabilizado um ponto para cada atividade realizada com independência, classificando os idosos em três níveis funcionais. O total de seis pontos significa independência para atividades de vida diária; quatro pontos, dependência parcial; e dois pontos, muito dependente. A dependência nas AVDs constitui-se como um marcador de maiores níveis de comprometimento funcional.

Foi realizada análise descritiva dos dados utilizando o programa estatístico Stata, utilizando estatísticas descritivas básicas, como: frequência, média, mediana, desvio padrão e valores mínimo e máximo, com nível de significância estabelecido em $\alpha=0,05$.

Diante da necessidade de atender e garantir às exigências éticas e científicas fundamentais para a realização de estudos com seres humanos, o estudo foi encaminhado ao Comitê de Ética e Pesquisa (CEP) da Universidade Estadual de Montes Claros e aprovado sob o número do parecer: 3.812.628. Todos os aspectos éticos foram respeitados. A direção da ILPI pesquisada apoiou a realização do estudo. Todos os participantes foram previamente informados sobre os objetivos da pesquisa e sobre a preservação do anonimato, sendo que o consentimento dos participantes foi formalizado por meio da assinatura do Termo de Consentimento Livre e Esclarecido (TCLE).

\section{RESULTADOS}

Avaliação do grau de independência para desempenho de AVDs foi realizada com 24 indivíduos com 60 anos de idade ou mais, sendo 13 homens (54,2\%) e 11 mulheres (45,8\%). A idade variou entre 60 e 95 anos, a maioria dos sujeitos possuía idade acima de 80 anos (42\%, ou 10), seguindo-se a faixa de 70 e 79 anos (37\%, ou 9) e de 60 a 69 anos (21\%, ou 5). Quanto à escolaridade, houve predomínio de analfabetos (83\%) ou com baixa escolaridade $(16,7 \%)$.

Quanto aos vínculos familiares, $71 \%$ não possuíam filhos e $58 \%$ recebiam visitas. Verificou-se predominância de solteiros $(70,8 \%)$, seguida de viúvos $(20,8 \%)$, casados $(4,2 \%)$ e divorciados $(4,2 \%)$. A tabela 2 descreve os resultados encontrados em relação ao perfil sociodemográfico dos idosos (Tabela 1).

\begin{tabular}{|c|c|c|}
\hline Variável & $\mathbf{N}$ & $\%$ \\
\hline \multicolumn{3}{|l|}{ Sexo } \\
\hline Masculino & 13 & 54,2 \\
\hline Feminino & 11 & 45,8 \\
\hline \multicolumn{3}{|l|}{ Estado civil } \\
\hline Solteiro & 17 & 70,8 \\
\hline Casado & 1 & 4,2 \\
\hline Divorciado/Separado & 1 & 4,2 \\
\hline Viúvo & 5 & 20,8 \\
\hline \multicolumn{3}{|l|}{ Escolaridade } \\
\hline Analfabeto & 20 & 83,3 \\
\hline$\leq 4$ anos de estudo & 4 & 16,7 \\
\hline$>4$ anos de estudo & 0 & 0 \\
\hline \multicolumn{3}{|l|}{ Possui Filhos } \\
\hline Sim & 7 & 29,2 \\
\hline Não & 17 & 70,8 \\
\hline \multicolumn{3}{|l|}{ Recebe visitas } \\
\hline Sim & 14 & 58,3 \\
\hline Não & 10 & 41,7 \\
\hline Total & 100 & \\
\hline
\end{tabular}

Fonte: Dias FSS, et al., 2020

Por meio do Índice de Katz, é avaliado o desempenho do indivíduo em atividades de autocuidado, obedecendo a uma hierarquia de complexidade. A escala é composta por seis itens: alimentação, controle de esfíncteres, transferência, higiene pessoal, capacidade para se vestir e tomar banho (ANDRIOLO BNG, et al., 2016). O item que apresentou maior índice de independência foi alimentar-se (79,2\%), ao passo que vestirse foi o item com o maior índice de dependência (54,2\%). Nesse estudo, identificou-se que grande parcela dos idosos era independente para a realização de cinco dos seis itens da escala (Tabela 2). 
Tabela 2 - Descrição da realização das atividades de vida diária (índice de Katz). $\mathrm{n=24}$.

\begin{tabular}{lcc}
\hline Variável & $\mathbf{N}$ & $\%$ \\
\hline Banhar-se & 13 & 54,2 \\
$\quad$ Independente & 11 & 45,8 \\
Dependência completa & & \\
\hline Vestir-se & 11 & 45,8 \\
$\quad$ Independente & 13 & 54,2 \\
Dependência completa & & \\
\hline Uso do banheiro & 13 & 54,2 \\
\hline$\quad$ Independente & 11 & 45,8 \\
$\quad$ Dependência completa & & \\
\hline Tranferir-se & 13 & 54,2 \\
$\quad$ Independente & 11 & 45,8 \\
$\quad$ Dependência completa & & \\
\hline Continência & 13 & 54,2 \\
$\quad$ Independente & 11 & 45,8 \\
$\quad$ Dependência completa & & \\
\hline Alimentar-se & 19 & 79,2 \\
$\quad$ Independente & 5 & 20,8 \\
$\quad$ Dependência completa & $\mathbf{2 4}$ & - \\
\hline Total & & \\
\hline
\end{tabular}

Fonte: Dias FSS, et al., 2020.

\section{DISCUSSÃO}

A avaliação da capacidade funcional geriátrica apreende informações do perfil funcional dos idosos, que incluem a análise do desempenho nos cuidados pessoais, de mobilidade e também de interação social (WIBELINGER LM, 2015). Dessa forma, o desempenho atividades de vida diária é utilizado como parâmetro para determinar o estado funcional do indivíduo, sendo amplamente utilizado pelos profissionais da área de saúde, pois colabora no planejamento de intervenções que são necessárias, prevenindo incapacidades.

Verificou-se que nessa instituição há predominância do sexo masculino entre os idosos residentes, resultado também encontrado em estudo realizado por Santiago LM, et al. (2016), conduzido com 760 idosos institucionalizados nas regiões sudeste e centro-oeste do Brasil, em que mais da metade era do sexo masculino (52,6\%).

Quanto à parentalidade, a maioria dos idosos era solteira $(70,8 \%)$ e $20,8 \%$ eram viúvos, bem a maioria não possuía filhos, correspondendo a $70,8 \%$. Os resultados obtidos quanto aos aspectos familiares dos idosos é semelhante à pesquisa realizada no Litoral Norte do Rio Grande do Sul, em que a maioria dos idosos não possuía companheiros nem filhos, sendo $46,7 \%$ viúvos, $31,6 \%$ eram solteiros e $63,3 \%$ não possuíam filhos (GÜTHS JFS, et al, 2017). Destaca-se que esses fatores podem estar relacionados à institucionalização, devido à ausência de um familiar próximo para cuidar do idoso, evidenciando a hipótese de que a ausência suporte social ou familiar pode ser uma das causas da institucionalização. (SANTIAGO LM, et al., 2016)

Em estudo realizado por Santiago LM, et al. (2016), verificou-se que mais da metade dos idosos de Campo Grande e de Cuiabá, e de 20 a $30 \%$ dos idosos do Sudeste não eram alfabetizados, corroborando os resultados encontrados nesse estudo, com predomínio de analfabetos (83,3\%), seguido de baixa escolaridade $(14,7 \%)$.

Sobre as visitas, a grande maioria dos idosos declarou receber visitas. Os achados desta pesquisa são corroborados pelo estudo de Güths JFS, et al. (2017) visto que $75 \%$ dos idosos recebiam visitas. $O$ recebimento de visitas pelos idosos institucionalizados demonstra que a família e/ou voluntários mantém relações de proximidade com o residente, descaracterizando a questão do abandono do idoso na ILPI. Ressalta-se que de acordo com os autores, as visitas são muito importantes, tendo em vista que contribui 
para que o mesmo apresente melhores condições de saúde, adaptação e participação nas atividades propostas (FLUETTI MT, et al., 2018).

A capacidade funcional é definida com a habilidade física e mental para manter a independência para a plena realização de uma tarefa pelo indivíduo. Assim, o idoso é classificado como dependente quando precisa totalmente de outra pessoa para a realização da atividade; parcialmente dependente é aquele que necessita de auxílio de outra pessoa para o desempenho da atividade; e independente é quando o idoso desenvolve a atividade sem nenhum auxílio.

Nesse estudo, na avaliação do desempenho das atividades básicas de vida diária, utilizando o Índice de Katz, entre os três níveis de independência, constatou-se que a maioria dos idosos era independente (54,2\%), seguida dos dependentes $(37,5 \%)$ e dos parcialmente dependentes $(8,3 \%)$.

De acordo com os dados obtidos com a aplicação do questionário Índice de Katz, os resultados do presente estudo sugerem que os idosos apresentam maior independência para a realização de cinco, dentre os seis itens da escala: banhar-se: 54,0\%; uso do banheiro: 54,2\%; transferir-se: 54,2\%; continência urinária e fecal: 54,2\%; alimentar-se: 79,2\%. Apenas na atividade de vestir-se, a maior parte da população foi dependente. Esse achado foi diverso do encontrado por Andriolo BNG, et al (2016), cujo estudo identificou a incontinência como a atividade de maior índice de dependência. A capacidade de se alimentar sozinho foi atividade com maior porcentagem de independência $(79,2 \%)$ e vestir-se, a atividade de menor frequência de independência $(45,8 \%)$.

No item "vestir-se" que a dependência foi identificada em 56,2\% dos idosos, sendo predominante no sexo feminino e nos acamados. Referente ao "uso do banheiro" verificou-se que 54\% eram independentes para realizar a atividade, dentre os dependentes (75\%) não deambulavam. Tendo em vista que a mobilidade é essencial para execução de outras atividades, observou-se que a maior parte dos idosos utiliza o andador como auxílio para locomoção. Conforme Güths JFS, et al. (2017), o uso desse dispositivo pode favorecer a independência funcional do idoso.

Em "transferência", observou-se que 54\% eram independentes, ou seja, conseguiam subir e descer da cama assim como sentar-se e levantar-se da cadeira sem assistência (podia utilizar algum auxílio como bengala ou andador) com predominância no sexo masculino $(58,3 \%)$. Com relação à "continência" verificouse que $54 \%$ tinham o completo controle das eliminações urinária e intestinal, $46 \%$ eram incontinentes ou apresentavam ocasionalmente perda urinária e intestinal. Quanto à continência, quinta atividade avaliada pelo Índice, houve concordância com estudo realizado por Leite et al. (2020), mostrando que a maior parte dos idosos são independentes.

O último item avaliado do Índice de KATZ, "alimentar-se", constatou-se que a maioria (79,2\%) conseguia se alimentar sem assistência, 20,8\% recebiam assistência e dentre os dependentes predominaram os idosos do sexo masculino. Em estudo realizado por Oliveira Neto J, et al. (2019), com 25 idosas institucionalizadas residentes em casas de repouso na cidade de Fortaleza-CE, a grande maioria das participantes (64\%) apresentou-se com grau de independência para realização das atividades de vida diária, corroborando os resultados desse estudo. No entanto, em relação às atividades, identificou-se que os idosos apresentaram maior grau de dependência em higiene pessoal e transferência, enquanto que nesse estudo verificou-se maior dependência em vestir-se.

Em relação ao grau de dependência relacionado aos grupos etários, observou-se que a maior parte dos idosos dependentes possuía idade superior a 80 anos (60\%). Nesse sentido, observa-se que as limitações da população idosa tornam-se mais evidentes quando atinge a faixa etária dos 80 anos de idade, com tendência a apresentar maiores dificuldades na realização das atividades da vida diária, acarretando perda da autonomia e independência (CASTRO VC e CARREIRA L, et al., 2015). Entretanto, de acordo com Oliveira Neto J, et al. (2019) a dependência não configura um estado permanente, mas trata-se de um processo dinâmico que pode ser modificado, prevenido ou limitado, por meio de serviços qualificados e comprometidos com a assistência ao idoso. 
Cabe ressaltar que, muitas vezes os resultados encontrados não condizem com a ideia que o idoso tem de si, pois em estudo de cunho qualitativo, desenvolvido por Colussi EL, et al. (2019), foram levantadas as percepções de idosos acerca do envelhecimento, e esses associaram esse processo à sentimentos de autonomia e independência, apresentando um imaginário social ativo, digno e saudável, o que talvez possa não ser a realidade encontrada pela equipe de saúde. Dessa forma, torna-se necessária a atuação da equipe de saúde na mensuração dos graus de dependência, para assim desenvolver um cuidado individualizado, realmente pautado na necessidade desses idosos, sendo que a utilização de escalas validadas, como a de Katz, pode direcionar o julgamento clínico e facilitar a tomada de decisão.

Uma análise global da condição de saúde do idoso, por meio de escalas validadas como a Escala de Katz, é condição essencial para um cuidado de qualidade, centrado nas particularidades de cada indivíduo. A utilização dessas escalas deve ser uma constante nas instituições que acolhem idosos, a fim de que a equipe de saúde possa identificar e atuar de maneira efetiva na manutenção da capacidade funcional dos idosos atendidos. Portanto, as instituições têm um papel fundamental na evolução do grau de dependência do idoso, podendo estimular ou limitar a realização de atividades dessa população.

Apenas o uso de escalas, no entanto, não é garantia da implementação de intervenções voltadas à prevenção do declínio funcional em idosos, sendo fulcral a combinação dos resultados da escala com o planejamento e implementação do cuidado. Assim, é de extrema importância que as instituições de longa permanência estejam estruturadas, tanto física quanto organizacionalmente, para avaliarem rotineiramente 0 quadro do residente, com a finalidade de acompanhar e impedir o declínio funcional do idoso e que essa prática seja guiada por protocolos assistenciais que estimulem o autocuidado e que sejam direcionados ao enfrentamento dos principais problemas que acometem os idosos (VIEIRA VAS, et al., 2018).

Além disso, as ILPI devem promover atividades de lazer, esportivas, culturais e educacionais, atuando efetivamente na promoção da saúde e prevenção de agravos, sendo que tais intervenções devem considerar as escolhas dos indivíduos acerca de suas vidas, numa perspectiva emancipatória e de interação social (CASTRO VC, CARREIRA L, 2015; SILVA RS, et al., 2019).

Quanto aos profissionais de saúde, estes devem compreender a multiplicidade e a complexidade dos fatores relacionados ao envelhecimento e institucionalização, com elaboração de planos de cuidados e intervenções de acordo com as características pessoais e sociais dos idosos residentes nessas instituições, reconhecendo as particularidades de cada idoso, com estímulo à independência e autonomia (SILVA RS, et al., 2019).

Como estudos futuros, elencamos a necessidade de identificar a presença e adoção de protocolos e planos de cuidados pela ILPI analisada, bem como a adequação dos mesmos à realidade da ILPI, no que tange aos recursos físicos, humanos e orçamentários, e às necessidades da população atendida.

\section{CONCLUSÃO}

Ao analisar os resultados desse estudo, conclui-se que residem predominantemente nessa instituição de longa permanência, idosos solteiros, que não possuem filhos, analfabetos ou de baixa escolaridade. Ressaltase, ainda, que essas características encontradas, muitas vezes, são consideradas como fatores de risco para institucionalização. Na avaliação do desempenho das atividades básicas de vida diária, utilizando o Índice de Katz, entre os três níveis de independência, constatou-se que a maioria dos idosos era independente. A maior parte dos idosos independentes desta instituição era do sexo masculino e com idade inferior a 80 anos. Dentre os seis itens, os resultados sugerem que os idosos possuem maior independência para a realização de cinco tarefas: banhar-se, ir ao banheiro, transferir-se, continência urinária e fecal e alimentar-se. Apenas na atividade de vestir-se, a maior parte da população foi dependente, sendo predominante no sexo feminino e nos acamados. Ressalta-se que os fatores que podem limitar a capacidade funcional dos idosos são variáveis para cada pessoa, tornando-se necessário um planejamento adequado por parte dos profissionais e da gestão das ILPI, de modo a garantir um maior grau de independência e qualidade de vida a este seguimento populacional. 


\section{REFERÊNCIAS}

1. ANDRIOLO BNG, et al. Avaliação do grau de funcionalidade em idosos. Revista da Sociedade Brasileira de Clínica Médica, 2016; 14(3): 139-44.

2. ANVISA. Agência Nacional de Vigilância Sanitária. RDC nํ283, de 26 de setembro de 2005. Regulamento técnico para o funcionamento das instituições de longa permanência para idosos. Brasília: ANVISA; 2005.

3. AZEVEDO LM, et al. Perfil sociodemográfico e condições de saúde de idosos institucionalizados. Rev. Bras. Pesq. Saúde, 2017; 19(3): 16-23.

4. CARRARA BS, ESPÍRITO SANTO, PMF. Old age institutionalized in postmodern times: the identity in parallel universe? Revista de Enfermagem UFPE on line [Internet], 2016; 10(5): 1672- 89.

5. CASTRO VC, CARREIRA L. Atividades de lazer e atitude de idosos institucionalizados: subsídios para a prática de enfermagem. Rev Lat Am Enfermagem [Internet], 2015; 23(2): 307-14 .

6. COLUSSI EL, et al. Percepções de idosos e familiares acerca do envelhecimento. Rev. bras. geriatr. gerontol., Rio de Janeiro, 2019; 22(1).

7. FLUETTI MT, et al. A síndrome da fragilidade em idosos institucionalizados. Rev. bras. geriatr. gerontol. [Internet], 2018; 21(1): 60-69.

8. GONÇALVES MJC, et al. A importância da assistência do enfermeiro ao idoso institucionalizado em instituição de longa permanência. Revista Científica de Enfermagem [Internet], 2015; 5(14): 12-8.

9. GUTHS, JFS, et al. Perfil sociodemográfico, aspectos familiares, percepção de saúde, capacidade funcional e depressão em idosos institucionalizados no Litoral Norte do Rio Grande do Sul, Brasil. Revista brasileira geriatria e gerontologia, 2017; 20(2): 175-185.

10. KAGAWA CA, CORRENTE JE. Análise da capacidade funcional em idosos do município de Avaré-SP: fatores associados. Revista Brasileira Geriatria e Gerontologia [Internet], 2015; 18(3): 577-86.

11. KATZ S, et al. Studies of illness in the aged. The index of ADL: a standardized measure of biological and psychosocial function. JAMA, 1963; 185(12): 914-9.

12. LEAL RC, et al. Efeitos do envelhecer: grau de dependência de idosos para as atividades da vida diária. Braz. J. of Develop., 2020; 6(7): 53931-53940.

13. LEITE AK, et al. Capacidade funcional do idoso institucionalizado avaliado pelo KATZ. Revista Enfermagem Atual in Derme, 2020; 91(29): 101-109.

14. OLIVEIRA NETO J, et al. Avaliação da funcionalidade em idosas institucionalizadas. Revista Desafios, 2019; 6(3): 306.

15. SANTIAGO LM, et al. Condições sociodemográficas e de saúde de idosos institucionalizados em cidades do sudeste e centro-oeste do Brasil. Geriatr Gerontol Aging, 2016; 10(2): 86-92.

16. SILVA RS, et al. Condições de saúde de idosos institucionalizados: contribuições para ação interdisciplinar e promotora de saúde. Cadernos Brasileiros de Terapia Ocupacional, 2019; 27(2): 345-356.

17. SOARES PPB, et al. Perception of purpose in life of elderly with depressive symptoms. Cogitare Enfermagem [Internet], 2015; 20(4): 670-74.

18. VIEIRA VAS, et al. Risco de lesão por pressão em idosos com comprometimento na realização de atividades diárias. Revista de Enfermagem do Centro-Oeste Mineiro, 2018; 8/2599.

19. WIBELINGER LM. Avaliação em fisioterapia geriátrica. In: WIBELINGER LM. Fisioterapia em Geriatria. 1. ed. Rio de Janeiro: Revinter, 2015; 1-37. 